\title{
The surface affinity of cations depends on both the cations and the nature of the surface
}

Cite as: J. Chem. Phys. 150, 044706 (2019); https://doi.org/10.1063/1.5065075

Submitted: 09 October 2018 . Accepted: 07 December 2018 . Published Online: 29 January 2019

Sudipta Das, Mischa Bonn (D), and Ellen H. G. Backus (D)

\section{COLLECTIONS}

Paper published as part of the special topic on Nonlinear Spectroscopy and Interfacial Structure and Dynamics Note: This article is part of the Special Topic "Nonlinear Spectroscopy and Interfacial Structure and Dynamics" in J. Chem. Phys.
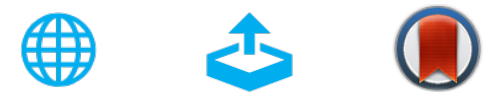

\section{ARTICLES YOU MAY BE INTERESTED IN}

Re-orientation of water molecules in response to surface charge at surfactant interfaces The Journal of Chemical Physics 151, 034703 (2019); https://doi.org/10.1063/1.5066597

Water structure at the interface of alcohol monolayers as determined by molecular dynamics simulations and computational vibrational sum-frequency generation spectroscopy

The Journal of Chemical Physics 150, 034701 (2019); https://doi.org/10.1063/1.5072754

Development of ultrafast broadband electronic sum frequency generation for charge dynamics at surfaces and interfaces

The Journal of Chemical Physics 150, 024708 (2019); https://doi.org/10.1063/1.5063458

Lock-in Amplifiers Find out more today

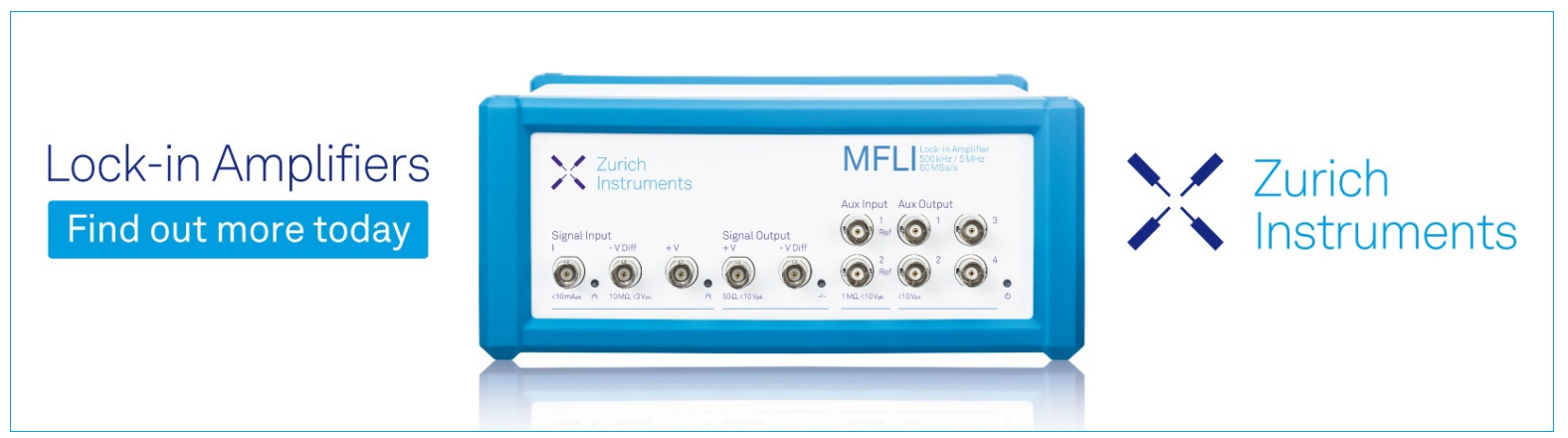




\title{
The surface affinity of cations depends on both the cations and the nature of the surface
}

\author{
Cite as: J. Chem. Phys. 150, 044706 (2019); doi: 10.1063/1.5065075 \\ Submitted: 9 October 2018 - Accepted: 7 December 2018 • \\ Published Online: 29 January 2019
}

Sudipta Das,' Mischa Bonn, ${ }^{1}$ (D) and Ellen H. G. Backus ${ }^{1,2, a)}$ (D)

\author{
AFFILIATIONS \\ ${ }^{1}$ Max Planck Institute for Polymer Research, Ackermannweg 10, 55128 Mainz, Germany \\ ${ }^{2}$ Department of Physical Chemistry, University of Vienna, Währinger Strasse 42, 1090 Vienna, Austria
}

Note: This article is part of the Special Topic "Nonlinear Spectroscopy and Interfacial Structure and Dynamics" in J. Chem. Phys.

a) Author to whom correspondence should be addressed: backus@mpip-mainz.mpg.de

\begin{abstract}
Specific ion effects at interfaces are important for a variety of thermodynamic properties of electrolyte solutions, like surface tension and the phase behavior of surfactants. We report the relative surface affinity of $\mathrm{Na}^{+}$and $\mathrm{D}_{3} \mathrm{O}^{+}$at both the $\mathrm{D}_{2} \mathrm{O}$-air and the sodium dodecyl sulfate (surfactant)-covered $\mathrm{D}_{2} \mathrm{O}$ surface by studying the alignment of interfacial $\mathrm{D}_{2} \mathrm{O}$, using vibrational sum frequency generation spectroscopy. The surface propensity of ions is found to be a function of both the nature of the ion and the nature of the surface. Specifically, for the charged, surfactant-covered interface, $\mathrm{Na}^{+}$has a higher affinity than $\mathrm{D}_{3} \mathrm{O}^{+}$. In contrast, $\mathrm{D}_{3} \mathrm{O}^{+}$has a higher affinity than $\mathrm{Na}^{+}$at the air- $\mathrm{D}_{2} \mathrm{O}$ interface. The relative surface affinity of cations thus depends on both details of the cation and the type of interface.
\end{abstract}

Published under license by AIP Publishing. https://doi.org/10.1063/1.5065075

\section{INTRODUCTION}

Ion specificity is important in various biochemical processes that frequently occur at the interface of water and lipid membranes of surfactants such as counterion dictated adsorption of surfactants at air-solution interfaces. ${ }^{7}$ It is proposed that at the water-air interface covered with charged organic monolayers, the coulomb interaction dictates the specific ion adsorption..$^{2,3}$ On the contrary, properties such as ion polarizability, ${ }^{4}$ ion size, ${ }^{5}$ dispersion forces, and hydration energy ${ }^{6}$ have been proposed to be dictating the ion adsorption at the neat water-air interface. It is indeed possible that different factors dominate specific ion adsorption at different interfaces. However, though water is an integral part of aqueous interfaces, the role of water itself in determining the ion specificity in the presence and absence of charged organic monolayers is not well understood. Therefore, it is important to understand the ion-specific effects on interfacial water organization in the presence of different types of ions and surfactants.

Surfactants are typically amphiphilic, owing to their characteristic nonpolar alkyl chains and polar head groups. They can adsorb at both polar (aqueous) and non-polar interfaces. At and beyond their critical micelle concentration (CMC), surfactants form a closely packed monolayer at the interface of water with air. The polar head groups directly interact with the water molecules and reduce the surface tension of water.

Due to this adsorption ability, surfactants are widely used in various industrial and biological applications, often in combination with salts and acids. The combination of surfactants and ions is also ubiquitous in the environment. In seawater, for instance, surfactants further interact with other chemicals and affect various physiochemical properties such as surface adsorption of these chemicals. ${ }^{7}$ Amongst different surfactants, those with charged head groups are abundant and interact strongly with water and aqueous electrolyte solutions. ${ }^{8,9}$ Surface tension studies, radiochemical studies, and simulations indicate that the counterions compete for surface adsorption at the water surface covered with charged surfactants. 2,3,10 In mixtures of monovalent cations, cations with smaller hydrated radius (i.e., higher charge density) tend to replace those with larger hydrated radius. Studies on charged lipid-covered aqueous interfaces have shown that the 
counterion concentration next to the lipid monolayer is much higher than in the subphase, ${ }^{11}$ as predicted by the GouyChapman model. ${ }^{12}$ The counterions screen the charge of the head groups and thereby influence the way interfacial water molecules align and interact with the surfactant/lipid head groups.

The resulting properties of such charged lipid/surfactant covered interfaces thus depend on the interplay of the surface charge, the presence of the counterions, and the water molecules. ${ }^{13}$ While this general understanding of charged interfaces exists, the details of how the structure of such charged surfaces depends on the type and concentration of counterions in solution are a priori unknown. To the best of our knowledge, the effect of protons on the alignment of water at such interfaces has not been reported, to date. This is surprising, considering the ubiquity of protons and their importance for countless physiochemical phenomena including protein (un-)folding and ion and charge transport through biological cell membranes.

To study the effect of protons on the alignment of water at a surfactant-covered aqueous surface, we have investigated a monolayer of sodium dodecyl sulfate ( $\mathrm{SDS}, \mathrm{R}-\mathrm{O}-\mathrm{SO}_{3}{ }^{-}$) on a $\mathrm{D}_{2} \mathrm{O}$ subphase containing excess $\mathrm{D}_{3} \mathrm{O}^{+}$. We have compared the results to those obtained from a subphase containing $\mathrm{Na}^{+}$and to the results from the same subphases without the surfactant, i.e., at the $\mathrm{D}_{2} \mathrm{O}$-air interface. As shown in Fig. 1(a), SDS has a negatively charged head-group and $\mathrm{Na}^{+}$as a counterion. It is a widely used component in, for example, shampoos, detergents, and toothpastes. As such, aqueous solutions of SDS containing various electrolytes are abundant in the environment. MD simulations ${ }^{14}$ have shown that for an SDS-covered water surface, SDS-head groups are hydrated and a significant population of $\mathrm{Na}^{+}$is present within these hydration shells. These $\mathrm{Na}^{+}$bridge different surfactant head groups and thereby stabilize the self-assembled monolayer (SAM). As mentioned above, here, we compare the interaction of $\mathrm{D}_{3} \mathrm{O}^{+}$and $\mathrm{Na}^{+}$with SDS. We determine the surface affinity of both ions by measuring the extent of water alignment, i.e., the extent of noncentrosymmetric organization of water, by vibrational Sum Frequency Generation (SFG).

SFG has been widely used to study the behavior of water in contact with surfactant and lipid monolayers ${ }^{15-18}$ and the bare water-air interface of aqueous electrolyte solutions. ${ }^{19-21}$ In SFG spectroscopy, a broadband IR pulse, spatiotemporally overlapped with a narrowband $800 \mathrm{~nm}$ pulse, impinges the sample. When the IR light is resonant with a molecular vibration, the SFG response is enhanced, resulting in a strong sum frequency signal. Centrosymmetric media like bulk water have zero SFG response, owing to the selection rules of the method. At the interface, however, the centrosymmetry is broken, resulting in an SFG signal from the interfacial water. This preferential alignment of water molecules is higher in the presence of charges at the surface like a monolayer of ionic surfactants. As a first order approximation, a more highly charged surface results in more aligned water molecules, giving rise to a higher SFG signal. At such surfactant-covered water surfaces, counterions present in the subphase can screen the charge and reduce the SFG intensity. Thus by monitoring the

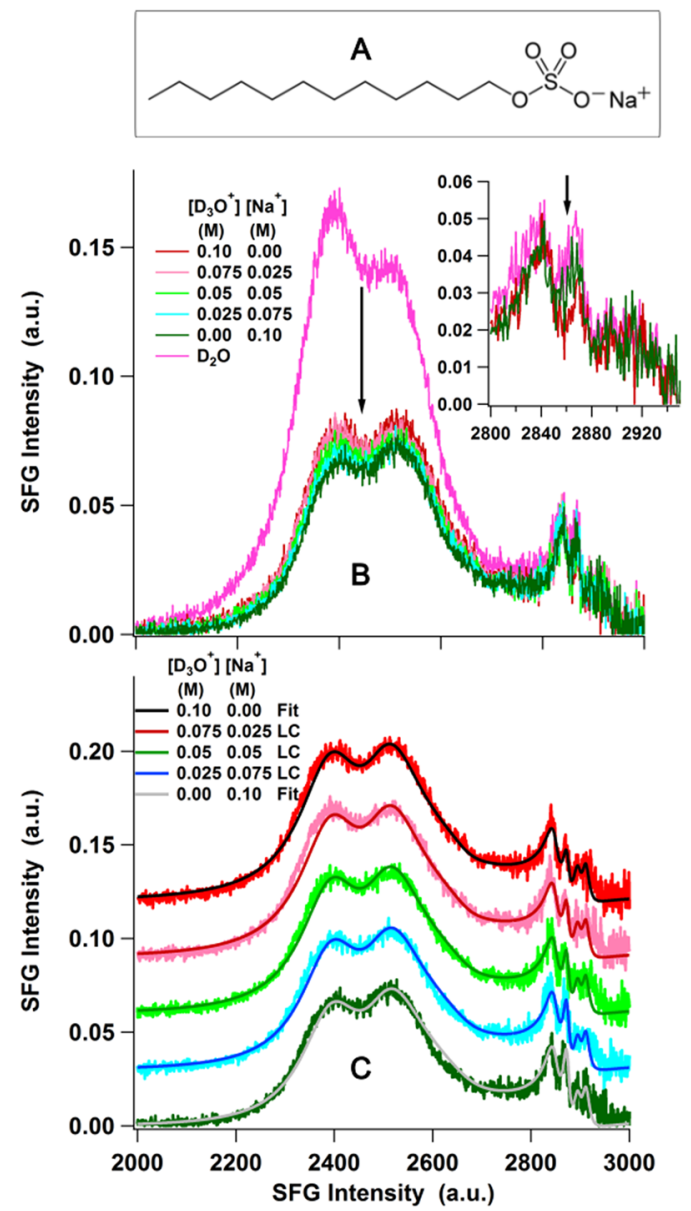

FIG. 1. (a) The chemical structure of sodium dodecyl sulfate (SDS). (b) SFG spectra in SSP polarization of an SDS-SAM on pure $\mathrm{D}_{2} \mathrm{O}$ (pink) and at different deuteron to sodium concentration ratios. The total concentration of cations is $0.1 \mathrm{M}$ in all cases. (c) Comparison of the spectra from (b) with the square of the weighted linear combinations (LC) of the $\chi^{(2)}$ responses of solutions containing $\left[\mathrm{Na}^{+}\right]=\left[\mathrm{D}_{3} \mathrm{O}^{+}\right]=0.1 \mathrm{M}$. The colors for experimental spectra in panels (b) and (c) are the same and are represented only in panel (b). The legends in panel (c) represent the colors of the fits and reconstructed spectra. The inset in panel (b) shows the zoomed-in $\mathrm{CH}$ stretch region, where a marked decrease in the symmetric $\mathrm{CH}_{3}$ intensity (highlighted by the arrow) is observed in the presence of $\mathrm{D}_{3} \mathrm{O}^{+}$.

SFG intensity as a function of the concentration of excess charges, we can quantify the relative surface affinity of $\mathrm{Na}^{+}$ and $\mathrm{D}_{3} \mathrm{O}^{+}$. Note that the correlation between the degree of water alignment and SFG signal intensity breaks down at low ionic strength. 22,23

We find that at the SDS covered aqueous interface, the relative interfacial affinity of $\mathrm{Na}^{+}$is higher than that of $\mathrm{D}_{3} \mathrm{O}^{+}$. At the bare water-air interface, the affinity order is the opposite. The relative surface affinity of counterions is thus interface dependent. Moreover, the order of the alkyl chain of the SDSSAM is lower in the presence of $\mathrm{D}_{3} \mathrm{O}^{+}$than in the presence of $\mathrm{Na}^{+}$indicating that the order of the SAM also depends on the type and concentration of the counterion present. 


\section{RESULTS AND DISCUSSIONS}

Figure 1(b) shows the SFG intensity in SSP polarization (S- for SFG and VIS, P- for IR) as a function of infrared frequency, recorded from the surface of a $12 \mathrm{mM}$ SDS solution in $\mathrm{D}_{2} \mathrm{O}$ without any excess ion and with co-containing $\mathrm{Na}^{+}$ and $\mathrm{D}_{3} \mathrm{O}^{+}$at different concentration ratios with a total cation concentration of $0.1 \mathrm{M}$. The pink curve shows the SFG intensity in the absence of any excess ions, and the red and the dark green in the presence of $0.1 \mathrm{M} \mathrm{D}_{3} \mathrm{O}^{+}$and $\mathrm{Na}^{+}$, respectively. The other three curves are responses in the presence of co-existing $\mathrm{Na}^{+}$and $\mathrm{D}_{3} \mathrm{O}^{+}$, where the total cation concentration is fixed at $0.1 \mathrm{M}$. Each spectrum has a broad response that extends from $\sim 2100 \mathrm{~cm}^{-1}$ to $\sim 2700 \mathrm{~cm}^{-1}$ and three narrower responses between $\sim 2800 \mathrm{~cm}^{-1}$ and $\sim 3000 \mathrm{~cm}^{-1}$. The broad feature is the vibrational response of the O-D groups located near the monolayer of SDS. As discussed in detail in Ref. 15, there are two sub-ensembles of OD groups at this interface which vibrate at $\sim 2390 \mathrm{~cm}^{-1}$ and $\sim 2510 \mathrm{~cm}^{-1}$. The OD groups that vibrate at $\sim 2510 \mathrm{~cm}^{-1}$ are hydrogen bonded to the head groups of SDS $\left(\mathrm{R}-\mathrm{O}-\mathrm{SO}_{3}{ }^{-}\right)$, and the ones that vibrate at $\sim 2390 \mathrm{~cm}^{-1}$ are not directly in contact with $-\mathrm{O}-\mathrm{SO}_{3}{ }^{-}$ groups, yet aligned under the influence of their electric field. The relatively sharp peaks at $\sim 2846 \mathrm{~cm}^{-1}, \sim 2870 \mathrm{~cm}^{-1}$, and $\sim 2910-2980 \mathrm{~cm}^{-1}$ are different $\mathrm{C}-\mathrm{H}$ vibrations of the alkyl chain of SDS.

The SFG intensity in the presence of both $0.1 \mathrm{M} \mathrm{D}_{3} \mathrm{O}^{+}$and $\mathrm{Na}^{+}$is significantly lower in the OD stretch region than in the absence of any excess ions in the solution. As predicted by the Gouy-Chapman model ${ }^{12}$ and shown by simulations, ${ }^{14}$ the $\mathrm{Na}^{+}$concentration near the charged monolayer head groups is extremely high even in the absence of any excess ions in the solution. Addition of excess $\mathrm{Na}^{+}$enhances the counterion concentration near the surface resulting in an increased charge screening and a concomitant reduction of the aligned water $\left(\mathrm{D}_{2} \mathrm{O}\right)$ molecules, thus reducing the SFG intensity. The SFG intensity along the OD stretch region gradually increases with increasing $\mathrm{D}_{3} \mathrm{O}^{+}$to $\mathrm{Na}^{+}$bulk concentration ratio. It indicates that the preferential alignment of $\mathrm{D}_{2} \mathrm{O}$ molecules near the charged head groups increases with the replacement of $\mathrm{Na}^{+}$by $\mathrm{D}_{3} \mathrm{O}^{+}$. This, in turn, indicates that the concentration of cations at the SDS- $\mathrm{D}_{2} \mathrm{O}$ interface decreases with increasing relative bulk concentration of $\mathrm{D}_{3} \mathrm{O}^{+} . \mathrm{D}_{3} \mathrm{O}^{+}$apparently adsorbs slightly less than $\mathrm{Na}^{+}$at the interface.

This observation can be explained by considering the ion radii of the cations and the implicit size restrictions imposed by the finite dimension of the electrical double layer (EDL) near the charged interface. Previous studies have already pointed out that smaller univalent cations are preferred within the EDL. 2,3,10 A larger number of smaller ions can remain within a constrained volume causing a better charge neutralization, thereby lowering the Gibbs free energy. Following this argument, our results thus indicate that $\mathrm{D}_{3} \mathrm{O}^{+}$should have a larger ion radius than $\mathrm{Na}^{+}$. X-ray and neutron diffraction studies indeed support this notion. The inter-nuclear distance between the central oxygen atom of $\mathrm{H}_{3} \mathrm{O}^{+}$and the oxygen atom of its nearest neighbor is $\sim 0.252 \mathrm{~nm}$ in $\mathrm{HCl}$ solution. For $\mathrm{DCl}$, it is even larger: $\sim 0.288 \mathrm{~nm}$. For sodium, however, both simulations and diffraction methods consistently report that the nuclear distance between the sodium ion and the nucleus of oxygen of the nearest $\mathrm{H}_{2} \mathrm{O}$ is $\sim 0.235 \mathrm{~nm} .^{24}$

Since these SFG responses arise from $\mathrm{D}_{2} \mathrm{O}$ molecules strongly aligned due to the electrostatic field of the SDS head groups, they possibly involve both pure $\chi^{(2)}$ and $\chi^{(3)}$ driven responses. ${ }^{17,22,23,25}$ In the present work, we focus on the relative changes in the intensities in the presence of $\mathrm{D}_{3} \mathrm{O}^{+}$and $\mathrm{Na}^{+}$. Therefore, we treat these intensities as an effective $\chi^{(2)}$ response, and we make no distinction between relative contributions of $\chi^{(2)}$ and $\chi^{(3)}$. The Debye length decreases from $1 \mu \mathrm{m}$ in the SDS covered pure $\mathrm{D}_{2} \mathrm{O}$ surface to $1 \mathrm{~nm}$ in SDS covered $\mathrm{D}_{2} \mathrm{O}$ containing $0.1 \mathrm{M} \mathrm{D}_{3} \mathrm{O}^{+}$and/or $\mathrm{Na}^{+}$(see the details in the supplementary material). Possibly the SFG response from the pure $\mathrm{D}_{2} \mathrm{O}$ case is a combined pure $\chi^{(2)}$ and $\chi^{(3)}$ response, while the SFG response in the presence of $0.1 \mathrm{M}$ excess ions is dominated by the $\chi^{(2)}$ contribution.

Not only the preferential organization of interfacial $\mathrm{D}_{2} \mathrm{O}$ but also the organization of the monolayer seems to be different for $\mathrm{D}_{3} \mathrm{O}^{+}$and $\mathrm{Na}^{+}$. The $\mathrm{CH}$ stretch modes, simultaneously measured in the experiments, provide information about the alkyl chain conformation. In particular, the ratio of intensities of the $\mathrm{CH}_{2}$ and $\mathrm{CH}_{3}$ symmetric stretch modes are known to serve as an order parameter of the alkyl chain.

The presence of $0.1 \mathrm{M} \mathrm{Na}^{+}$does not affect the SDS conformation: it changes the water response substantially, but not the intensities of both the $\mathrm{CH}_{2}$ symmetric stretch $\left(2846 \mathrm{~cm}^{-1}\right)$ and the $\mathrm{CH}_{3}$ symmetric stretch $\left(2870 \mathrm{~cm}^{-1}\right)$ modes of the SDS monolayer [see the inset in Fig. 1(b)]. In contrast, the presence of $0.1 \mathrm{M} \mathrm{D}_{3} \mathrm{O}^{+}$causes a $\sim 50 \%$ reduction in the $\mathrm{CH}_{3}$ symmetric stretch intensity. Since the SDS concentration $(12 \mathrm{mM})$ is for all $\mathrm{Na}^{+}$and $\mathrm{D}_{3} \mathrm{O}^{+}$concentrations studied here above the CMC of SDS, it is unlikely that the surface coverage changes significantly. The reduction in the $\mathrm{CH}$ vibration responses, therefore, indicates that the presence of $\mathrm{D}_{3} \mathrm{O}^{+}$causes a disordering of the SAM alkyl chain.

The $\mathrm{Na}^{+}$concentration next to the SAM head groups is very high, due to the Coulomb interactions, even in the absence of any excess ions in the solution. ${ }^{12}$ In the presence of $0.1 \mathrm{M}$ excess $\mathrm{Na}^{+}$in the sub-phase, the order of SAM alkyl chains hardly differs from that of neat $\mathrm{D}_{2} \mathrm{O}$. On the contrary, in the presence of $0.1 \mathrm{M} \mathrm{D}_{3} \mathrm{O}^{+}$, the SAM is disordered compared to that of neat $\mathrm{D}_{2} \mathrm{O}$, indicating that the directional characters of hydrogen bonding between the head groups and the counterions, in addition to the Coulomb interaction, are apparently important in determining the order of the SAM. The two cations are geometrically very different: $\mathrm{D}_{3} \mathrm{O}^{+}$has a "tripod" structure, ${ }^{26}$ as opposed to the spherical shape of $\mathrm{Na}^{+}$. As a result, the ability of those two cations to act as bridges between different head groups is undoubtedly different. Second, although the pKa of SDS in bulk- $\mathrm{H}_{2} \mathrm{O}$ is -1.5 , the surface $\mathrm{pKa}$ is expected to be $\sim 0$, based on reports regarding similar systems. ${ }^{11,27}$ Therefore, at a bulk $\mathrm{D}_{3} \mathrm{O}^{+}$concentration of $0.1 \mathrm{M}$, i.e., $\mathrm{pH}=1, \sim 10 \%$ of the SDS molecules should be protonated. The protonation of SDS molecules reduces the repulsion amongst the head groups, which is also expected to affect alkyl chain arrangement. Nevertheless, the effects of the presence of $0.1 \mathrm{M} \mathrm{D}_{3} \mathrm{O}^{+}$in the subphase on the preferential 
alignment of $\mathrm{D}_{2} \mathrm{O}$ molecules and the alkyl chains of the SAM at the interface are slightly different than in the presence of $0.1 \mathrm{M} \mathrm{Na}^{+}$. The $\mathrm{D}_{2} \mathrm{O}$ molecules are preferentially more aligned, but the alkyl chains are preferentially less aligned in the presence of $0.1 \mathrm{M} \mathrm{D}_{3} \mathrm{O}^{+}$compared to that of $0.1 \mathrm{M} \mathrm{Na}^{+}$. Both effects occur simultaneously.

Evidently, $\mathrm{Na}^{+}$and $\mathrm{D}_{3} \mathrm{O}^{+}$perturb the noncentrosymmetric order of interfacial $\mathrm{D}_{2} \mathrm{O}$ and the packing order of SDS-SAM to a different extent. Therefore, an interesting question is, whether all these effects are (non-)additive when more than one type of cation coexist in the solution. If the effects are additive with respect to their bulk concentrations then the intensity from a solution, containing a mixture of cations, should be equal to the square of the linear combinations of the $\chi^{(2)}$ response from the solution containing only one or the other cation. If the effects are additive with respect to their surface concentrations, it requires precise knowledge of their surface concentration which is not known.

Since calculating mixtures of the spectra as a linear combination of the response of the pure solutions requires knowledge of the $\chi^{(2)}$, we have quantitatively analyzed the data by fitting with an established procedure. According to this procedure, the SFG signal is proportional to the square of the second-order nonlinear susceptibility $\chi^{(2)}$ of the sample and intensity of both incoming IR and Visible (VIS) light,

$$
\mathrm{I}_{\mathrm{SFG}} \propto\left|\chi^{(2)}\right|^{2} \mathrm{I}_{\mathrm{VIS}} \mathrm{I}_{\mathrm{IR}}
$$

The second order nonlinear susceptibility $\chi^{(2)}$ is a sum of a non-resonant contribution $\chi_{\mathrm{NR}}^{(2)}$ (consisting of an amplitude $\mathrm{A}_{\mathrm{NR}}$ and a phase $\phi_{\mathrm{NR}}$ ) and resonant contribution(s) $\chi_{\mathrm{R}}^{(2)}$. Each $\chi_{R}^{(2)}$ is expressed in terms of a Lorentzian line shape with area $A_{n}$, central frequency $\omega_{n}$, and bandwidth $\Gamma_{n}$ (half width at half maximum)

$$
\left|\chi^{(2)}\right|^{2}=\left|\chi_{\mathrm{NR}}^{(2)}+\chi_{\mathrm{R}}^{(2)}\right|^{2}=\left|\mathrm{A}_{\mathrm{NR}} e^{i \phi_{\mathrm{NR}}}+\frac{\mathrm{A}_{n}}{\omega_{n}-\omega_{\mathrm{IR}}+i \Gamma_{n}}\right|^{2} .
$$

The spectra recorded for the SDS-covered pure aqueous $\mathrm{Na}^{+}$ and $\mathrm{D}_{3} \mathrm{O}^{+}$solutions are fitted with eight resonant peaks (See Table 1 in the supplementary material for assignment details). The bandwidths and peak positions of all the bands as well as the non-resonant area and phase from this fit are subsequently interpolated to describe the intermediate spectra.

Figure 1(c) shows that the three spectra for co-existing $\mathrm{Na}^{+}$and $\mathrm{D}_{3} \mathrm{O}^{+}$can be described very well by the square of the linear combinations of the $\chi^{(2)}$ response (see the supplementary material) from pure $\mathrm{Na}^{+}$and pure $\mathrm{D}_{3} \mathrm{O}^{+}$, implying that the effect is simply additive.

The observation that $\mathrm{Na}^{+}$has higher surface affinity at the SDS-water interface is in contrast with the conclusion drawn for the electrolyte-air interface, for which, at very high concentrations $(\sim 1 \mathrm{M}), \mathrm{H}_{3} \mathrm{O}^{+}$has been found ${ }^{20,28-30}$ to have higher interfacial affinity than $\mathrm{Na}^{+}$. Here, we have monitored the modulation of interfacial $\mathrm{D}_{2} \mathrm{O}$ alignment at the air- $\mathrm{D}_{2} \mathrm{O}$ interface as a function of the bulk $\mathrm{D}_{3} \mathrm{O}^{+}$and $\mathrm{Na}^{+}$concentration ratio at $1 \mathrm{M}$ constant ionic strength using vibrational $\mathrm{SFG}$ spectroscopy. Unlike the SDS case presented above, the alignment of interfacial $\mathrm{D}_{2} \mathrm{O}$ at the air-water interface is enhanced by the presence of ions, and the enhancement of the SFG intensity is a measure of the surface affinity of an ion. By systematically changing the $\mathrm{Na}^{+}$and $\mathrm{D}_{3} \mathrm{O}^{+}$ion concentration ratio, and quantitatively monitoring the change in the SFG intensity, we have studied not only the relative surface affinity of the two ions but also the gradual evolution of interfacial water alignment under the influence of the relative concentration ratio of these two ions.

Figure 2(a) shows the SFG responses in SSP polarization at the $\mathrm{D}_{2} \mathrm{O}$-air interface, for pure $\mathrm{D}_{2} \mathrm{O}$ and in the co-presence of $\mathrm{Na}^{+}$and $\mathrm{D}_{3} \mathrm{O}^{+}$at different concentration ratios at $1 \mathrm{M}$ total ionic strength. As opposed to the SDS case, higher ionic concentration at the air- $\mathrm{D}_{2} \mathrm{O}$ interface enhances the preferential $\mathrm{D}_{2} \mathrm{O}$ alignment. To ensure an optimum signal to noise ratio, we have chosen here a $1 \mathrm{M}$ ionic strength.

Each spectrum consists of two main features: a sharp peak centered at $\sim 2700 \mathrm{~cm}^{-1}$ and a relatively broad feature that extends between $\sim 2000 \mathrm{~cm}^{-1}$ and $\sim 2600 \mathrm{~cm}^{-1}$. The sharp
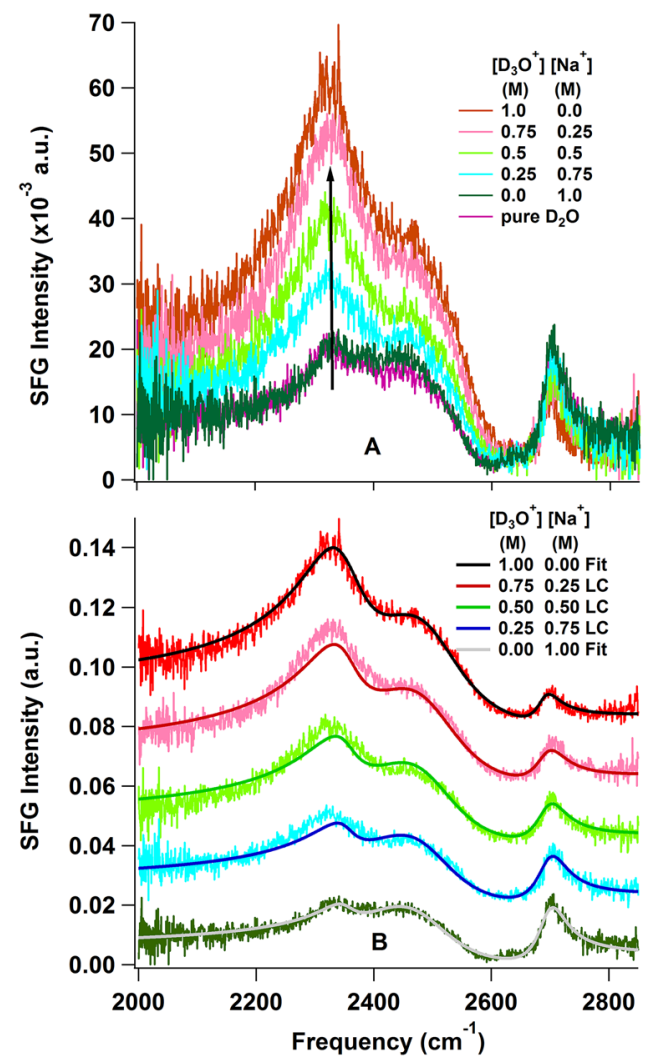

FIG. 2. (a) SFG spectra in SSP polarization of the $\mathrm{D}_{2} \mathrm{O}$-air interface for different sodium to deuterium concentration ratios. The total concentration of cations is $1 \mathrm{M}$ in all cases. (b) A comparison of original experimental spectra with the square of the weighted linear combinations of the $\chi^{(2)}$ responses of solutions containing $\left[\mathrm{Na}^{+}\right]=\left[\mathrm{D}_{3} \mathrm{O}^{+}\right]=1 \mathrm{M}$. The colors for the experimental spectra in panels (a) and (b) are the same and are represented only in panel (a). The legend in panel (b) represents the colors of the fits and reconstructed spectra. 
peak at $\sim 2700 \mathrm{~cm}^{-1}$ originates from the non-hydrogen bonded O-D oscillator which points away from the surface into the air. The broad feature extending between 2000 and $2600 \mathrm{~cm}^{-1}$ originates from OD groups with a broadly distributed $\mathrm{H}$-bond strength. Intra- and inter-molecular coupling among different OD vibrations causes the double-peak feature. ${ }^{31}$

The SFG intensity hardly changes when adding $1 \mathrm{M} \mathrm{Na}^{+}$to $\mathrm{D}_{2} \mathrm{O}$, meaning that the alignment of interfacial $\mathrm{D}_{2} \mathrm{O}$ molecules in the presence of $1 \mathrm{M} \mathrm{Na}^{+}$is almost identical to that of pure $\mathrm{D}_{2} \mathrm{O}: \mathrm{Na}^{+}$has no or very weak surface affinity towards the $\mathrm{D}_{2} \mathrm{O}$-air interface, as, apparently, does $\mathrm{Cl}^{-}$. In contrast, with increasing fraction of $\mathrm{D}_{3} \mathrm{O}^{+}$, the intensity around $\sim 2000-$ $2600 \mathrm{~cm}^{-1}$ increases and that around $2700 \mathrm{~cm}^{-1}$ decreases. The $\mathrm{D}_{3} \mathrm{O}^{+}$ions add charges to the $\mathrm{D}_{2} \mathrm{O}$-air interface, increasing the alignment of interfacial water. Since $\mathrm{D}_{3} \mathrm{O}^{+}$has a "tripod" structure and can make three strong hydrogen bonds with the neighboring $\mathrm{D}_{2} \mathrm{O}$ molecules, the preferential alignment of interfacial $\mathrm{D}_{2} \mathrm{O}$ molecules with their $\mathrm{D}$ atoms pointing towards bulk increases with increasing bulk $\mathrm{D}_{3} \mathrm{O}^{+}$concentration, thus resulting in an enhanced SFG intensity around the $\sim 2000-2600 \mathrm{~cm}^{-1}$ region. The reduction in intensity of the dangling OD peak can be a result of a real reduction in vibrational amplitude and/or a change in its angular distribution with respect to the surface normal. More studies to comment conclusively on these observations are ongoing. In any case, we find that, at the $\mathrm{D}_{2} \mathrm{O}$-air interface, $\mathrm{D}_{3} \mathrm{O}^{+}$indeed is more surface active than $\mathrm{Na}^{+}$at $1 \mathrm{M}$ concentration, in agreement with previous reports. ${ }^{32,33}$

Although, for pure $\mathrm{D}_{2} \mathrm{O}, \chi^{(3)}$ does not contribute to the SFG response, $\mathrm{D}_{2} \mathrm{O}$ containing ions certainly can. ${ }^{34-36}$ The increase in the SFG response in Fig. 2(a) is, therefore, possibly caused by simultaneous changes in pure $\chi^{(2)}$ along with changes in $\chi^{(3)}$ driven responses. In this study, we are interested only in relative changes in the total SFG intensity, and we therefore consider, as mentioned above, the combined effects as changes in an effective $\chi^{(2)}$ response.

Figure 2(b) shows the experimental SFG spectra in the presence of $1 \mathrm{M} \mathrm{Na}^{+}$and $\mathrm{D}_{3} \mathrm{O}^{+}$along with the fitted spectra (details of the fits are given in the supplementary material). Panel (b) also shows the experimental SFG spectra in the co-presence of $\mathrm{Na}^{+}$and $\mathrm{D}_{3} \mathrm{O}^{+}$along with the corresponding squared $\chi^{(2)}$ spectra constructed with linear combinations of the $\chi^{(2)}$ response of $1 \mathrm{M} \mathrm{D}_{3} \mathrm{O}^{+}$and $\mathrm{Na}^{+}$solutions obtained by fitting. The linear combinations significantly differ from the experimental spectra specifically around $2350 \mathrm{~cm}^{-1}$, meaning that the effects are not additive, unlike in the case of SDS. It qualitatively proves that apparently, the surface concentration of adsorbed $\mathrm{D}_{3} \mathrm{O}^{+}$does not increase linearly with its bulk concentration.

\section{CONCLUSION}

In summary, we have reported the relative interfacial affinity of two ubiquitous cations, $\mathrm{Na}^{+}$and $\mathrm{D}_{3} \mathrm{O}^{+}$(equivalent to $\mathrm{H}_{3} \mathrm{O}^{+}$in $\mathrm{H}_{2} \mathrm{O}$ ), and their effects on interfacial water organization at both the water-air and the surfactant-covered aqueous solution interface using SFG spectroscopy. Due to ionized head groups $\left(-\mathrm{O}-\mathrm{SO}_{3}{ }^{-}\right)$of $\mathrm{SDS}$, the interface is negatively charged. Both cations screen this surface charge, yet the screening ability depends slightly on the charge and radius of the cation. The surface propensity of $\mathrm{Na}^{+}$is marginally higher than that of $\mathrm{D}_{3} \mathrm{O}^{+}$. Moreover, the order of the alkyl chain of SDS also depends on the type of ions in the solution: $\mathrm{D}_{3} \mathrm{O}^{+}$makes the alkyl chains more disordered than $\mathrm{Na}^{+}$. Also these two effects are additive with respect to the co-existence of the two ions.

In contrast, at the nominally neutral air-aqueous solution interface, $\mathrm{D}_{3} \mathrm{O}^{+}$has much higher surface propensity than $\mathrm{Na}^{+}$. As such, the surface propensity of $\mathrm{Na}^{+}$and $\mathrm{D}_{3} \mathrm{O}^{+}$at the SDS-covered water surface is opposite to that at the airwater interface. However, the surface propensity and the ability to perturb the interfacial $\mathrm{D}_{2} \mathrm{O}$ alignment in the waterair interface is significantly non-additive with respect to co-existence of the two cations. Apparently, the surface adsorption of $\mathrm{D}_{3} \mathrm{O}^{+}$is not linearly related with its bulk concentration at the air $-\mathrm{D}_{2} \mathrm{O}$ interface. The ion-specific effect is larger for the nominally neutral air-water interface than for the charged SDS- $\mathrm{D}_{2} \mathrm{O}$ interface, where screening of that charge is, to a first approximation, independent of details of the cation.

\section{SUPPLEMENTARY MATERIAL}

The supplementary material contains the details of the experimental setup, fitting parameters for the fits shown in Figs. 1 and 2, the equation used for linear combinations of the fitted spectra shown in Figs. 1(b) and 2(b), and the Debye length and surface charge density.

\section{ACKNOWLEDGMENTS}

The authors thank M. Knecht for purifying the SDS samples and M. Deiseroth, C. Bernhard, S. Pezzotti, D.R. Galimberti, M.-P. Gaigeot, M. Grechko, and J. Hunger for fruitful discussions. This work was funded by a DFG grant (No. BA $5008 / 3)$.

\section{REFERENCES}

${ }^{1}$ T. Gilányi, I. Varga, and R. Mészáros, "Specific counterion effect on the adsorption of alkali decyl sulfate surfactants at air/solution interface," Phys. Chem. Chem. Phys. 6(17), 4338-4346 (2004).

${ }^{2}$ P. M. Biesheuvel and M. van Soestbergen, "Counterion volume effects in mixed electrical double layers," J. Colloid Interface Sci. 316(2), 490-499 (2007).

${ }^{3}$ V. L. Shapovalov and G. Brezesinski, "Breakdown of the Gouy-Chapman model for highly charged Langmuir monolayers: Counterion size effect," J. Phys. Chem. B 110(20), 10032-10040 (2006).

${ }^{4}$ L. X. Dang, "Computational study of ion binding to the liquid interface of water," J. Phys. Chem. B 106(40), 10388-10394 (2002).

${ }^{\mathbf{5}}$ Y. Levin, "Polarizable ions at interfaces," Phys. Rev. Lett. 102(14), 147803 (2009).

${ }^{6}$ K. D. Collins, G. W. Neilson, and J. E. Enderby, "Ions in water: Characterizing the forces that control chemical processes and biological structure," Biophys. Chem. 128(2), 95-104 (2007).

${ }^{7}$ E. Guzmán, E. Santini, A. Benedetti, F. Ravera, M. Ferrari, and L. Liggieri, "Surfactant induced complex formation and their effects on the interfacial properties of seawater," Colloids Surf., B 123, 701-709 (2014). 
${ }^{8}$ A. M. Mankowich, "The energetics of surfactant adsorption at the air-water interface," J. Am. Oil Chem. Soc. 43(11), 615-619 (1966).

${ }^{9} \mathrm{~S}$. Karakashev, R. Tsekov, and E. Manev, "Adsorption of alkali dodecyl sulfates on air/water surface," Langmuir 17(17), 5403-5405 (2001).

${ }^{10}$ J.-S. Sin, K.-I. Kim, H.-C. Pak, and C.-S. Sin, "Effect of orientational ordering of water dipoles on stratification of counterions of different size in multicomponent electrolyte solution near charged surface-a mean field approach," Electrochim. Acta 207, 237-246 (2016).

${ }^{11}$ A. Kundu, S. Yamaguchi, and T. Tahara, "Evaluation of $\mathrm{pH}$ at charged lipid/water interfaces by heterodyne-detected electronic sum frequency generation," J. Phys. Chem. Lett. 5(4), 762-766 (2014).

${ }^{12} \mathrm{~J}$. N. Israelachvili, "Electrostatic forces between surfaces in liquids," in Intermolecular and Surface Forces, 3rd ed., edited by J. N. Israelachvili (Academic Press, San Diego, 2011), Chap. 14, pp. 291-340.

${ }^{13}$ L. B. Dreier, Y. Nagata, H. Lutz, G. Gonella, J. Hunger, E. H. G. Backus, and M. Bonn, "Saturation of charge-induced water alignment at model membrane surfaces," Sci. Adv. 4(3), eaap7415 (2018).

${ }^{14} \mathrm{M}$. Chen, X. Lu, X. Liu, Q. Hou, Y. Zhu, and H. Zhou, "Specific counterion effects on the atomistic structure and Capillary-waves fluctuation of the water/vapor interface covered by sodium dodecyl sulfate," J. Phys. Chem. C 118(33), 19205-19213 (2014)

${ }^{15}$ R. A. Livingstone, Y. Nagata, M. Bonn, and E. H. G. Backus, "Two types of water at the water-surfactant interface revealed by time-resolved vibrational spectroscopy," J. Am. Chem. Soc. 137(47), 14912-14919 (2015).

${ }^{16}$ Y.-C. Wen, S. Zha, C. Tian, and Y. R. Shen, "Surface $\mathrm{pH}$ and ion affinity at the alcohol-monolayer/water interface studied by sum-frequency spectroscopy," J. Phys. Chem. C 120(28), 15224-15229 (2016).

${ }^{17}$ D. E. Gragson, B. M. McCarty, and G. L. Richmond, "Ordering of interfacial water molecules at the charged air/water interface observed by vibrational sum frequency generation," J. Am. Chem. Soc. 119(26), 6144-6152 (1997).

${ }^{18}$ D. E. Gragson, B. M. McCarty, and G. L. Richmond, "Surfactant/water interactions at the air/water interface probed by vibrational sum frequency generation," J. Phys. Chem. 100(34), 14272-14275 (1996).

${ }^{19}$ C. S. Tian, N. Ji, G. A. Waychunas, and Y. R. Shen, "Interfacial structures of acidic and basic aqueous solutions," J. Am. Chem. Soc. 130(39), 13033-13039 (2008).

${ }^{20}$ L. M. Levering, M. R. Sierra-Hernandez, and H. C. Allen, "Observation of hydronium ions at the air-aqueous acid interface: Vibrational spectroscopic studies of aqueous HCI, HBr, and HI," J. Phys. Chem. C 111(25), 8814-8826 (2007).

${ }^{21}$ E. A. Raymond and G. L. Richmond, "Probing the molecular structure and bonding of the surface of aqueous salt solutions," J. Phys. Chem. B 108(16), 5051-5059 (2004).

${ }^{22}$ G. Gonella, C. Lütgebaucks, A. G. F. de Beer, and S. Roke, "Second harmonic and sum-frequency generation from aqueous interfaces is modulated by interference," J. Phys. Chem. C 120(17), 9165-9173 (2016).
${ }^{23}$ J. Schaefer, G. Gonella, M. Bonn, and E. H. G. Backus, "Surface-specific vibrational spectroscopy of the water/silica interface: Screening and interference," Phys. Chem. Chem. Phys. 19(25), 16875-16880 (2017).

${ }^{24}$ Y. Marcus, "Ionic radii in aqueous solutions," Chem. Rev. 88(8), 1475-1498 (1988).

${ }^{25}$ Y.-C. Wen, S. Zha, X. Liu, S. Yang, P. Guo, G. Shi, H. Fang, Y. R. Shen, and C. Tian, "Unveiling microscopic structures of charged water interfaces by surface-specific vibrational spectroscopy," Phys. Rev. Lett. 116(1), 016101 (2016).

${ }^{26} \mathrm{~F}$. Giberti and A. A. Hassanali, "The excess proton at the air-water interface: The role of instantaneous liquid interfaces," J. Chem. Phys. 146(24), 244703 (2017).

${ }^{27} \mathrm{D}$. Wenger, "Acid-base behaviour of short-chain fatty acids on aqueous surfaces using $\mathrm{x}$-ray photoelectron spectroscopy, nuclear magnetic resonance and molecular dynamics," M.S. thesis, Uppsala University, 2015. ${ }^{28}$ D. Liu, G. Ma, L. M. Levering, and H. C. Allen, "Vibrational spectroscopy of aqueous sodium halide solutions and air-liquid interfaces: Observation of increased interfacial depth," J. Phys. Chem. B 108(7), 2252-2260 (2004).

${ }^{29} \mathrm{~T}$. Ishiyama and A. Morita, "Molecular dynamics analysis of interfacial structures and sum frequency generation spectra of aqueous hydrogen halide solutions," J. Phys. Chem. A 111(38), 9277-9285 (2007).

${ }^{30} \mathrm{~T}$. Ishiyama and A. Morita, "Molecular dynamics study of gas-liquid aqueous sodium halide interfaces. I. Flexible and polarizable molecular modeling and interfacial properties," J. Phys. Chem. C 111(2), 721-737 (2007).

${ }^{31} \mathrm{~J}$. Schaefer, E. H. G. Backus, Y. Nagata, and M. Bonn, "Both interand intramolecular coupling of $\mathrm{O}-\mathrm{H}$ groups determine the vibrational response of the water/air interface," J. Phys. Chem. Lett. 7(22), 4591-4595 (2016).

${ }^{32}$ W. Hua, D. Verreault, and H. C. Allen, "Relative order of sulfuric acid, bisulfate, hydronium, and cations at the air-water interface," J. Am. Chem. Soc. 137(43), 13920-13926 (2015).

${ }^{33} \mathrm{P}$. B. Petersen and R. J. Saykally, "Evidence for an enhanced hydronium concentration at the liquid water surface," J. Phys. Chem. B 109(16), 79767980 (2005).

${ }^{34}$ S. Pezzotti, D. R. Galimberti, Y. R. Shen, and M.-P. Gaigeot, "Structural definition of the BIL and DL: A new universal methodology to rationalize non-linear $\chi^{(2)}(\omega)$ SFG signals at charged interfaces, including $\chi^{(3)}(\omega)$ contributions," Phys. Chem. Chem. Phys. 20(7), 5190-5199 (2018).

${ }^{35} \mathrm{~S}$. Pezzotti and M.-P. Gaigeot, "Spectroscopic BIL-SFG invariance hides the chaotropic effect of protons at the air-water interface," Atmosphere 9(10), 396 (2018).

${ }^{36}$ S. Pezzotti, D. R. Galimberti, and M.-P. Gaigeot, "2D H-bond network as the topmost skin to the air-water interface," J. Phys. Chem. Lett. 8(13), 31333141 (2017). 